\title{
Texture Boundary Detection - A Structural Approach
}

\author{
Wen Wen Richard J. Fryer \\ Machine Perception Research Group, \\ Department of Computer Science \\ University of Strathclyde, Glasgow G1 1XH, United Kingdom
}

\begin{abstract}
Perception of different textures is caused by differences in distribution of properties of texture elements. However, in practice it is difficult to extract useful texture elements, especially from natural images in which texture elements exist at various scales. To extract texture elements of all sizes a multiscale approach is unavoidable. This paper describes a multiscale method, based on measurements in a Laplacian-ofGaussian scale-space, to extract texture elements. Histograms are used to describe the distribution of properties of extracted texture elements in a region. The edge significance at a pixel reflects the difference in the histograms of the regions surrounding the pixel. High edge significance pixels constitute the texture boundaries. Performance of the approach is shown for various natural textured images.
\end{abstract}

\section{Introduction}

Psychological studies of texture perception suggest that texture segmentation is based on the first order statistics of properties of texture elements (texels) such as orientation, size and contrast [1,2]. Most psychological experiments are carried out by employing synthetic textured images in which the texels are symbols such as line segments, rectangles and crossings. However, these kinds of texel are not available in a natural scene. In this paper, we propose a multiscale method to extract texels from natural textured images, followed by detection of texture boundaries on the basis of the distribution of properties of extracted texels

\section{Texel extraction}

A number of approaches to extracting texels have been proposed $[3,4,5,6]$. These methods are a single scale processes. This leads to two disadvantages. Firstly, it needs 
human interference such setting scale of operators and threshold values. Secondly, even when the scale of the operators is set, it may not produce satisfactory results since texels usually have a large range of sizes especially in a natural scene. Indeed, Blostein and Ahuja [7] have taken into account various sizes of texel, texels extracted by their approach are disk-like. Thus, their approach may not be suitable for segmentation since texture segmentation is not only based on size of texture element but also on other features such as orientation and length.

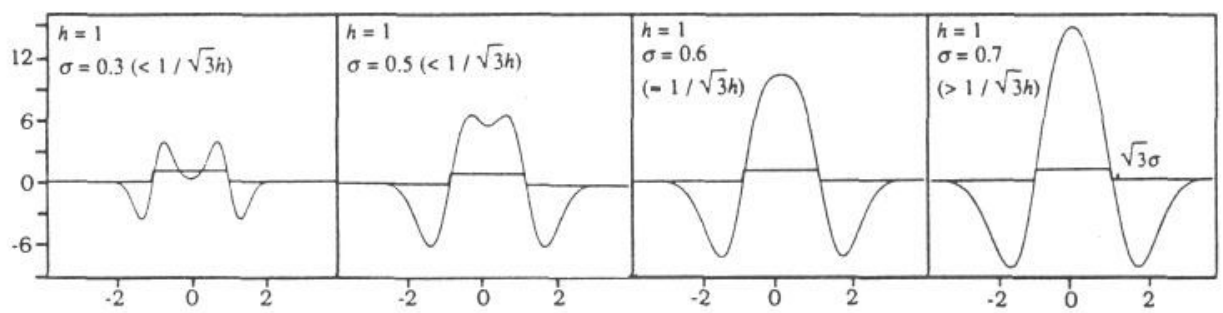

Figure 1: Convolution of a convex ridge (which is assumed to be a profile of a light bar) with $\nabla^{2} G$ of different $\sigma$ values.

The proposed multiscale approach for texel extraction is based on the analysis of the convolution a ideal bar image with a Laplacian of a Gaussian, $\nabla^{2} G$. The image of a bar is defined by:

$$
I(x, y)= \begin{cases}l & |x| \leq h \\ l_{2} & \text { elsewhere }\end{cases}
$$

where $h$ is the half width of the bar, and

$$
\nabla^{2} G(x, y)=\frac{2 \sigma^{2}-\left(x^{2}+y^{2}\right)}{\sigma^{4}} e^{-\left(x^{2}+y^{2}\right) / 2 \sigma^{2}} .
$$

We have shown [8] that a maximum (minimum) occurs on the main axis of a light (dark) bar in the convolved image when $\sigma>h / \sqrt{3}$. Otherwise, the maximum (or minimum) occurs off the axis of the bar and its location is a function of the value $\sigma$. Figure 1 demonstrates that maximum locations of convolution of a convex ridge vary with various $\sigma$ values. Based on this, the location of the axis of a bar can be located at extrema which do not shift at least in two convolved images with different $\sigma$ values. If a pixel locates at the axis of a bar and there is an extremum at this pixel in the convolved image with an $\sigma$, the width $h$ of the bar must be less than $\sqrt{3} \sigma$ (derived from $\sigma>h$ $/ \sqrt{3}$ ). Thus, the other part of the bar can be found by examining the pixels within the area $2 \sqrt{3} \sigma$ by $2 \sqrt{3} \sigma$ centred at the location of the extreme in the convolved image. If the 
pixel value is higher (lower) that zero, it is one of the elements of the light (dark) bar (cf. Figure 1).

The implementation of the texel extraction algorithm is summarized as follows.

(1) Convolving the image by a set of Laplacian of Gaussian masks with sizes $3 \times 3,5 \times 5$, $7 \times 7,9 \times 9,11 \times 11,13 \times 13,15 \times 15,17 \times 17,19 \times 19,21 \times 21$, and $23 \times 23$ pixels. The $\sigma$ value for a particular mask $(w \times w)$ is $w / 7$.

(2) The main axis of a light texel (an area brighter than its surrounding) is located at local maxima which occur at the same location in at least two convolved images. For the location of the main axis of a dark texel (an area darker than its surrounding), we mark the local minima occurring at the same location in at least two convolved images.

(3) At each location on the main axis, the smallest $\sigma$ value under which the extreme occurs at this location in the convolved image is used to define an examination area $2 \sqrt{3} \sigma$ by $2 \sqrt{3} \sigma$ centred at the extreme. Within the area, all pixels in the convolved image larger (smaller) than zero are marked as pixels of a texel.
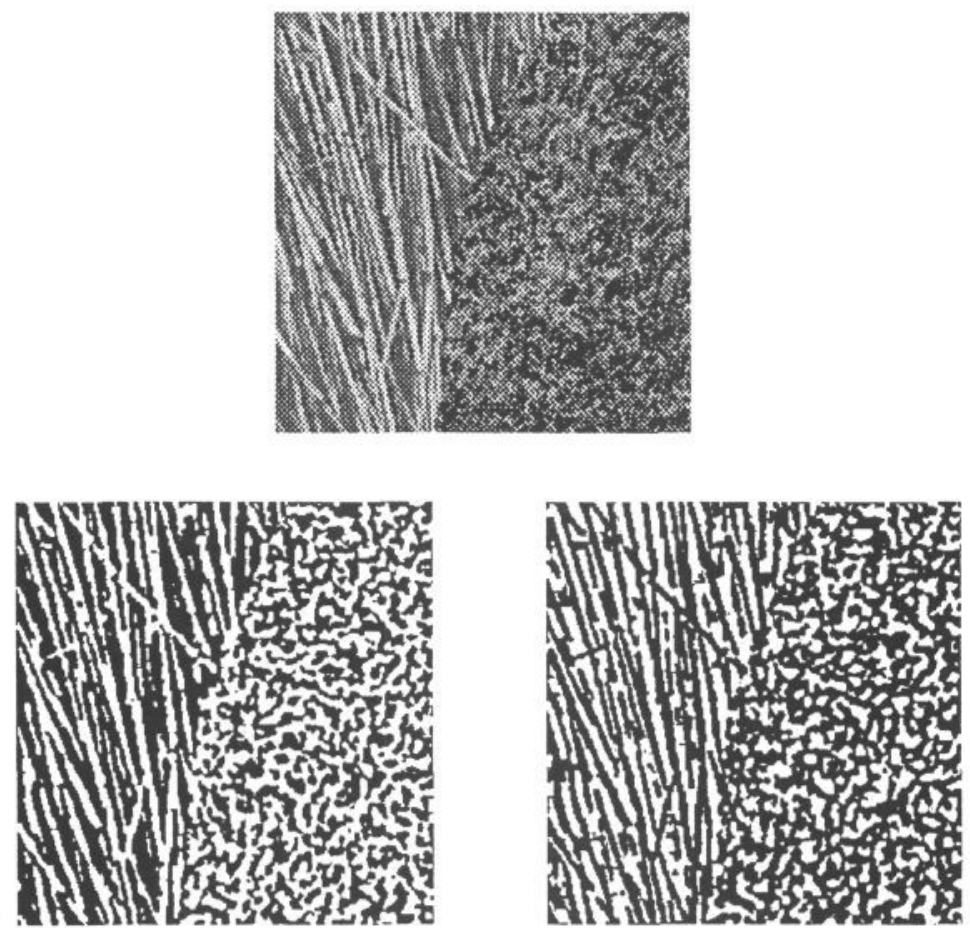

Figure 2: An image (top) of straw and grass; and the extracted light (bottom left) and dark texels(bottom right). 

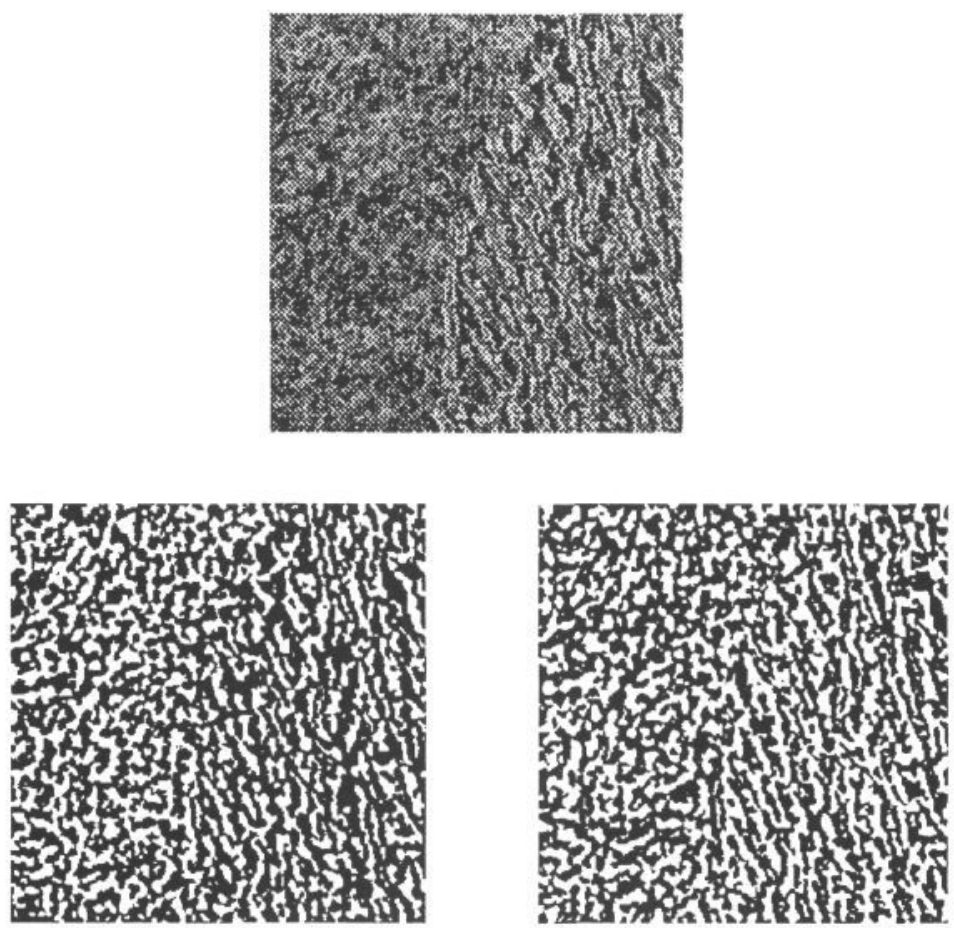

Figure 3: An image (top) of grass and leather; and the extracted light (bottom left) and dark texels(bottom right).

Figure 2 and 3 illustrate the performance of the texel extraction algorithm. The texel images are binary with texel in white and background in black. As demonstrated, the algorithm reliably detects texture elements in a large range of sizes and of various shapes from natural images without any human interference. It is insensitive to the lighting condition of the images.

\section{Texture boundary detection}

Properties such as orientation, length, size and contrast, of texels extracted in previous section are used as texture features. A texture edge corresponds to a discontinuity in texture features. The significance of a texture edge at a pixel reflects the significance of differences in distribution of texture features in the regions surrounding the pixel. 


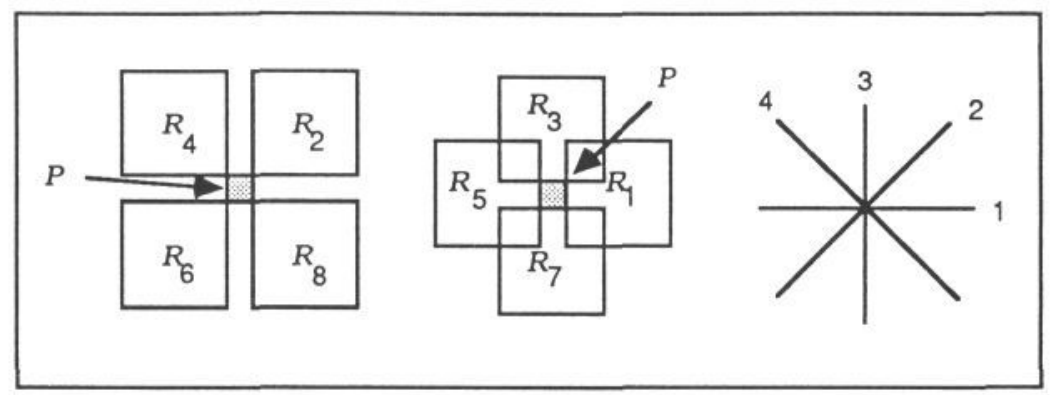

Figure 4: Pixel $P$, its surrounding eight regions $R_{1}, R_{2}, \ldots R_{8}$, and four directions $(1,2,3$, and 4$)$ across it.

Figure 4 illustrates a pixel, its surrounding eight regions $R_{1}, R_{2}, \ldots R_{8}$, and four directions across the pixel. Distribution of a feature $F$ in the eight regions is described by a set of histograms, $H_{1}, H_{2}, \ldots$, and $H_{8}$, of the feature, respectively. A histogram $H$ of feature $F$ in a region $R$ can be computed in the following way. The domain of $F$ is divided into a number of ranges (bins), $f_{1}, f_{2}, \ldots, f_{\mathrm{n}}$. An element of the histogram $H(i)$ is the number of texels within the region $R$ whose feature values fall into the corresponding range $f_{\mathrm{i}}$. The histograms are normalized to get the corresponding density functions $D_{i}, i=1,2, \ldots, 8$ :

$$
D_{i}(j)=\frac{H_{i}(j)}{\sum_{k=1}^{N} H_{i}(k)}
$$

where $H_{i}(j)$ is the value of the bin $j$ in the histogram $H_{i}$ and $N$ is the number of bins in a histogram. The difference between two regions across the pixel $P$ in a particular direction $i$ is calculated by their maximum density difference [6], i.e.,

$$
V_{i}(P \mid F)=\max _{j}\left\{\left|D_{i}(j)-D_{i+4}(j)\right|\right\} \quad j=1,2 \ldots N .
$$

The edge significance of pixel $P$ in the texture feature $F$ is the maximum difference among four pairs of bordering regions:

$$
S(P \mid F)=\max _{i}\left\{V_{i}(P \mid F)\right\} \quad i=1,2,3,4 .
$$

The combination edge significance of the pixel $P$ in multiple texture features is defined as the maximum of the weighted significance in each feature, that is:

$$
S(P)=\max _{l}\left\{C_{l} S\left(P \mid F_{l}\right)\right\} \quad l=1,2,3,4 .
$$


$C_{l}$ is the weighting factor for feature $F_{l}$ which correspond to feature characteristics: orientation, length, area and contrast. The value of $C_{l}$ is dynamically dependent on different visual tasks.

High edge significance pixels constitute the texture boundaries. Texture boundaries are located by a non-maximum suppression technique in the significance image, followed by a linking process.

Figure 5 and 6 are the results of boundaries found in the images (a) in Figure 2 and 3, respectively.

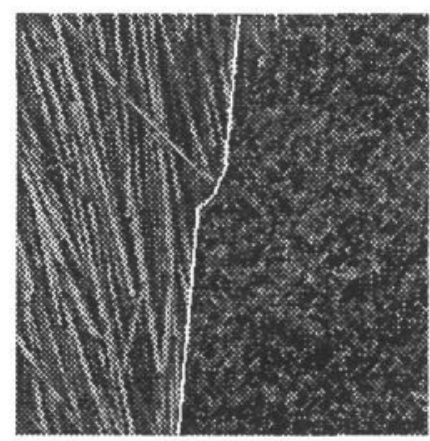

Figure 5: The detected boundaries from straw and grass image (Figure 2)

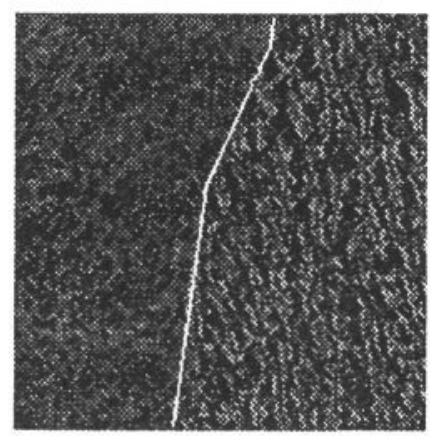

Figure 6: The detected boundaries from grass and leather image (Figure 3) 


\section{Conclusion}

Texture segmentation is based on differences in texture feature distribution. Most existing structural texture segmentation algorithms do not deal with the problem of how to extract texture elements with various sizes. In this paper, we present a multiscale method to extract texels. The implementation results show that the algorithm successfully extracts various sizes of texel from natural images without requiring externally supplied parameters such as a threshold. It is insensitive to the noise and lighting condition in the images. An algorithm is proposed to use the distributions of the properties of the extracted texels to distinguish different textures. The detected boundaries correspond to those which humans perceive except near the image boundary area.

\section{References}

[1] Beck, J. Texture segmentation, in Organization and Representation in Perception, Ed. J. Beck, Erlbaum, Hillsdale, New Jersey, 1982, pp.285-317.

[2] Julesz, B. and Bergen, J.R., Textons, the fundamental elements in preattentive vision and perception of textures, in Readings in Computer Vision, ed. M. A. Fischler and O. Firschein, Morgan Kaufmann Publishers, INC. 1987, pp 243- 256.

[3] Tomita, F., Shirai, Y. and Tsuji, S., Description of textures by a structural analysis, IEEE Tran. on Patt.Anal. Machine Intell. vol PAMI-4, pp 679-698, March 1987.

[4] Rearick, T.C, A texture of analysis algorithm inspired by a theory of preattentive vision, Proc. of Conf. on Computer Vision and Pattern Recognition, pp 312-317, 1985.

[5] Vilnrotter, E.M., Nevatia, R and Price K.E. Structure analysis of natural textures, IEEE Trans. on Patt. Anal. and Machine Intell., Vol. PAMI-8, No.1. Jan. 1986.

[6] Voorhees, H.L., and Poggio, T., Detecting textons and texture boundaries in natural images, in Proc. of First Int. Conf. on Computer Vision. London England, 8-11 June 1987, pp.250-258.

[7] Blostein, D. and Ahuja, N. A multiscale region detector, Comp. Vision, Graphics and Image Proc. 45, 22-41, 1989.

[8] Wen, W. and Fryer, R.J. Multiscale texture element detection, in press. 